\title{
GLOBAL DYNAMICS OF THE BUCKINGHAM'S TWO-BODY PROBLEM
}

\author{
JAUME LLIBRE ${ }^{1}$, CLAUDIA VALLS $^{2}$ AND CLAUDIO VIDAL ${ }^{3}$
}

\begin{abstract}
The Buckingham systems are the equations of motion of a two-body problem defined by the Hamiltonian

$$
\frac{1}{2}\left(p_{x}^{2}+p_{y}^{2}\right)+A e^{-B \sqrt{x^{2}+y^{2}}}-\frac{M}{\left(x^{2}+y^{2}\right)^{3}},
$$

where $A, B$ and $M$ are positive constants. This Hamiltonian and its corresponding angular momentum $p_{\theta}$ are two first integrals, independent and in involution. Let $I_{h}$ (respectively, $I_{c}$ ) be the set of points of the phase space on which $H$ (respectively, $p_{\theta}$ ) takes the value $h$ (respectively, $c)$. Since $H$ and $p_{\theta}$ are first integrals, the sets $I_{h}$ and $I_{h c}=I_{h} \cap I_{c}$ are invariant under the flow of the Buckingham systems. We characterize the global flow of these systems describing the foliation of the phase space by the invariant sets $I_{h}$, the foliation of $I_{h}$ by the invariant subsets $I_{h c}$, and the foliation of $I_{h c}$ by the flow of the system.
\end{abstract}

\section{Introduction AND STATEMENT OF THE MAIN RESUlts}

A simplification of the classical Lennard-Jones potential (see $[6,9])$ for studying the motion under the attractive or repulsive gravitational and intermolecular forces is the Buckingham potential

$$
U(x, y)=A e^{-B \sqrt{x^{2}+y^{2}}}-\frac{M}{\left(x^{2}+y^{2}\right)^{3}},
$$

introduced in 1928 for studying the state equation for gaseous helium, neon or argon, see [3]. Here $A, B$ and $M$ are positive parameters. More precisely, this potential describes the van der Waals energy in the interaction of two atoms at the distance $\sqrt{x^{2}+y^{2}}$, and the Pauli repulsion energy.

Recently the Buckingham potential has been studied in [10, 11, 12]. In these papers the authors studied their equilibria and their orbits using the McGehee coordinates and taking into account when the energy $H$ is negative, zero or positive.

2010 Mathematics Subject Classification. Primary 34A05. Secondary 34C05, 37C10. Key words and phrases. Buckingham equations, Hill regions, Global dynamics. 
In this paper we describe the global dynamics of the Buckingham's twobody problem, i.e. of the Hamiltonian

$$
H\left(x, y, p_{x}, p_{y}\right)=\frac{1}{2}\left(p_{x}^{2}+p_{y}^{2}\right)+A e^{-B \sqrt{x^{2}+y^{2}}}-\frac{M}{\left(x^{2}+y^{2}\right)^{3}} .
$$

We change to canonical polar coordinates (see page 154 of [8]) by setting

$$
x=r \cos \theta, y=r \sin \theta, p_{x}=p_{r} \cos \theta-\frac{p_{\theta}}{r} \sin \theta, p_{y}=p_{r} \sin \theta+\frac{p_{\theta}}{r} \cos \theta .
$$

In these new variables the Hamiltonian system is

$$
\begin{aligned}
\dot{r} & =\frac{\partial H}{\partial p_{r}}=p_{r} \\
\dot{\theta} & =\frac{\partial H}{\partial p_{\theta}}=\frac{p_{\theta}}{r^{2}}, \\
\dot{p_{r}} & =-\frac{\partial H}{\partial r}=A B e^{-B r}-\frac{6 M}{r^{7}}+\frac{p_{\theta}^{2}}{r^{3}}, \\
\dot{p_{\theta}} & =-\frac{\partial H}{\partial \theta}=0
\end{aligned}
$$

where

$$
H=\frac{1}{2}\left(p_{r}^{2}+\frac{p_{\theta}^{2}}{r^{2}}\right)+A e^{-B r}-\frac{M}{r^{6}} .
$$

The Hamiltonian $H$ and the angular momentum $p_{\theta}$ are two first integrals, independent and in involution. Hence the Hamiltonian system (1) is completely integrable in the sense of Liouville-Arnold, see for instance $[1,2,8]$.

If we denote by $\mathbb{R}^{+}$the open interval $(0, \infty)$, then the phase space of the Buckingham systems is $E=\mathbb{R}^{+} \times \mathbb{S}^{1} \times \mathbb{R}^{2}$ where $r \in \mathbb{R}^{+}, \theta \in \mathbb{S}^{1}$ and $\left(p_{r}, p_{\theta}\right) \in \mathbb{R}^{2}$. Since $H$ and $p_{\theta}$ are first integrals, the sets

$$
\begin{aligned}
I_{h} & =\left\{\left(r, \theta, p_{r}, p_{\theta}\right) \in E: H\left(r, \theta, p_{r}, p_{\theta}\right)=h\right\}, \\
I_{h c} & =\left\{\left(r, \theta, p_{r}, p_{\theta}\right) \in E: H\left(r, \theta, p_{r}, p_{\theta}\right)=h, p_{\theta}=c\right\},
\end{aligned}
$$

are invariant by the Hamiltonian flow of system (1), i.e. if a solution curve of system (1) has a point in $I_{h}$ or in $I_{h c}$ all the solution curve is contained into $I_{h}$ or $I_{h c}$, respectively.

The main results of this paper are the descriptions of the foliations of the phase space $E$ as union of the invariant sets $I_{h}$, the invariant sets $I_{h}$ by the union of the invariant subsets $I_{h c}$, and the subsets $I_{h c}$ by the flow of the Hamiltonian system.

These foliations provide a good description of the global dynamics of the Hamiltonian systems (1) when $K$ varies, where

$$
K=\left(\frac{7}{e B}\right)^{7}-\frac{6 M}{A B} .
$$

Here $e$ is the number $e=2.718282 \cdots$. 
The paper is organized as follows. In section 2 we recall Liouville-Arnold's theory about integrable Hamiltonian systems applied to the Buckhingham systems.

The Hill region, $R_{h}$, is the region of the position space where takes place the motion of all orbits having energy $h$. In section 3 we classify all the Hill regions for the Buckhingham systems, all the sets $I_{h c}$, and how these last sets foliate $I_{h}$.

\section{InTEGRABle HAMiltonian Systems}

In this section we apply the Liouville-Arnold Theorem to the integrable Hamiltonian systems (1). We recall that a flow defined on a subspace of the phase space is complete if its solutions are defined for all time.

Theorem 1 (Liouville-Arnold Theorem). The Hamiltonian system (1) with 2 degrees of freedom defined on the phase space $E$ has the Hamiltonian $H$ and angular momentum $p_{\theta}$ as two independent first integrals in involution. If $I_{h c} \neq \emptyset$ and $(h, c)$ is a regular value of the map $\left(H, p_{\theta}\right)$, then the following statements hold.

(a) $I_{h c}$ is a 2-dimensional submanifold of $E$ invariant under the flow of (1).

(b) If the flow on a connected component $I_{h c}^{*}$ of $I_{h c}$ is complete, then $I_{h c}^{*}$ is diffeomorphic either to the torus $\mathbb{S}^{1} \times \mathbb{S}^{1}$, or to the cylinder $\mathbb{S}^{1} \times \mathbb{R}$. We note that if $I_{h c}^{*}$ is compact (i.e. $I_{h c}^{*} \approx \mathbb{S}^{1} \times \mathbb{S}^{1}$ ), then the flow on it is always complete.

(c) Under the hypothesis (b) the flow on $I_{h c}^{*}$ is conjugated to a linear flow either on $\mathbb{S}^{1} \times \mathbb{S}^{1}$, or on $\mathbb{S}^{1} \times \mathbb{R}$.

For more details about Hamiltonian systems and the proof of the previous theorem see Abraham and Marsden [1] and Arnold [2, 4]. We remark that in general, under the assumptions of statement (b), $I_{h c}^{*}$ can also be diffeomorphic to the plane $\mathbb{R}^{2}$, but this is not the case for the Buckhingham systems, because their Hamiltonians do not depend on the variable $\theta$, and consequently the manifolds $I_{h c}$ must have a factor $\mathbb{S}^{1}$.

Note that the Liouville-Arnold Theorem for our Buckhingham systems does not provide information on the topology of the invariant sets $I_{h c}$ when $(h, c)$ is not a regular value of the map $\left(H, p_{\theta}\right)$, and how is the flow on these invariant sets, or how the invariant sets $I_{h c}$ foliate the energy sets $I_{h}$, or how the energy levels $I_{h}$ foliate the phase space $E$.

In this paper we solve all these questions for the Buckhingham systems. For a generic study of the invariant sets $I_{h c}$ for Hamiltonian systems of two degrees of freedom having a central potential, see [7]. 


\section{The TOPOLOGY of $I_{h}$ AND $I_{h c}$}

A point $\left(r, \theta, p_{r}, p_{\theta}\right) \in E$ is critical for the map $H: E \rightarrow \mathbb{R}$ if it is an equilibrium of system (1). A point of $E$ which is not critical is regular. The value $h \in \mathbb{R}$ is critical for the map $H: E \rightarrow \mathbb{R}$ if there is some critical point belonging to $H^{-1}(h)=I_{h}$. If $h \in \mathbb{R}$ is not critical, then $h$ is a regular value. It is well-known that if $h$ is a regular value of the map $H: E \rightarrow \mathbb{R}$, then $I_{h}$ is a 3-dimensional manifold, see for instance [5].

Since $r>0$ the critical points of $H$ must satisfy

$$
g(r)=r^{7} e^{-B r}-\frac{6 M}{A B}=0, \quad p_{r}=p_{\theta}=0 .
$$

So the set of critical points of $H$ is

$$
\begin{aligned}
\mathcal{C} & =\left\{(r, \theta, 0,0) \in E: g(r)=0 \text { and } \theta \in \mathbb{S}^{1}\right\} \\
& \approx\{(r, 0,0): g(r)=0\} \times \mathbb{S}^{1} .
\end{aligned}
$$

We need to study the zeroes of the function $g(r)$. Note that

$$
\frac{d g}{d r}=-e^{-B r} r^{6}(B r-7)
$$

and so $g$ has a maximum at $r=7 / B$ and

$$
g\left(\frac{7}{B}\right)=\left(\frac{7}{e B}\right)^{7}
$$

Note that the set of critical points $\mathcal{C}$ of $H$ is equal to

$$
\begin{aligned}
& \emptyset \quad \text { if } K<0, \\
& \left\{(7 / B, \theta, 0,0) \in E: \theta \in \mathbb{S}^{1}\right\} \quad \text { if } K=0, \\
& \left\{\left(r_{1}, \theta, 0,0\right) \in E: \theta \in \mathbb{S}^{1}\right\} \cup\left\{\left(r_{2}, \theta, 0,0\right) \in E: \theta \in \mathbb{S}^{1}\right\} \quad \text { if } K>0,
\end{aligned}
$$

where $r_{1}<7 / B<r_{2}$. Hence, the critical value is $-A e^{-7} / 6$ if $K=0$, and the values $-M / r_{1}^{6}+A e^{-B r_{1}}$ or $-M / r_{2}^{6}+A e^{-B r_{2}}$ if $K>0$.

Let $\pi: E \rightarrow \mathbb{R}^{+} \times \mathbb{S}^{1}$ be the natural projection from the phase space $E$ to the configuration space $\mathbb{R}^{+} \times \mathbb{S}^{1}$. Then for each $h \in \mathbb{R}$ the Hill region $R_{h}$ of $I_{h}$ is defined by $R_{h}=\pi\left(I_{h}\right)$. Therefore, setting

$$
f_{h}(r)=h+\frac{M}{r^{6}}-A e^{-B r}
$$

we have

$$
R_{h}=\left\{(r, \theta) \in \mathbb{R}^{+} \times \mathbb{S}^{1}: f_{h}(r) \geq 0\right\} \approx\left\{r \in \mathbb{R}^{+}: f_{h}(r) \geq 0\right\} \times \mathbb{S}^{1},
$$

where as usual $\approx$ means diffeomorphic to. Note that the Hill region $R_{h}$ is the region of the configuration space or position space where takes place the motion of all orbits having energy $h$.

Note that

$$
\lim _{r \rightarrow+\infty} f_{h}(r)=h \quad \text { and } \quad \lim _{r \rightarrow 0} f_{h}(r)=+\infty
$$


Moreover, when $K>0$ we have that $f_{h}(r)$ has a minimum and a maximum which we denote by $f_{1}$ and $f_{2}$, respectively.

We compute the energy levels $I_{h}$ in two different ways. The first way is more direct, and the second way allows additionally to deduce the foliation of $I_{h}$ by the invariant sets $I_{h c}$.

From the definition of $I_{h}$ we have that

$$
I_{h}=\bigcup_{(r, \theta) \in R_{h}} E_{(r, \theta)},
$$

where

$$
E_{(r, \theta)}=\left\{\left(r, \theta, p_{r}, p_{\theta}\right) \in E: p_{r}^{2}+\frac{p_{\theta}^{2}}{r^{2}}=2 f_{h}(r)\right\} .
$$

Clearly for each $(r, \theta)$ given the set $E_{(r, \theta)}$ is an ellipse, a point, or the empty set if the point $(r, \theta)$ belongs to the interior of $R_{h}$, to the boundary of $R_{h}$, or does not belong to $R_{h}$, respectively. Therefore, from (2), it follows easily the topology of $I_{h}$ according with the different values of $h$ and $K$.

Another way of computing again the invariant energy levels $I_{h}$ is using

$$
I_{h}=\left\{\left(r, \theta, p_{r}, p_{\theta}\right) \in E: H\left(r, \theta, p_{r}, p_{\theta}\right)=h\right\} \approx g^{-1}(h) \times \mathbb{S}^{1},
$$

where $g\left(r, p_{r}, p_{\theta}\right)=H\left(r, \theta, p_{r}, p_{\theta}\right)$.

If $h \in \mathbb{R}$ is a regular value of the map $g: \mathbb{R}^{+} \times \mathbb{R}^{2} \rightarrow \mathbb{R}$ and $g^{-1}(h) \neq \emptyset$, then $g^{-1}(h)$ is a surface of $\mathbb{R}^{+} \times \mathbb{R}^{2}$. It is easy to verify that the intersection of $g^{-1}(h)$ with $\left\{r=r_{0}=\right.$ constant $\}$, is an ellipse, a point, or the empty set according to $f_{h}\left(r_{0}\right)$ is positive, zero, or negative, respectively. So by studying the union of the ellipses or points of the form $g^{-1}(h) \cap\left\{r=r_{0}\right\}$ moving $r_{0}>0$, we obtain the sets $g^{-1}(h)$. Therefore, from (3), we calculate in a different way the topology of the energy levels $I_{h}$.

We note that knowing the sets $g^{-1}(h)$, from

$$
\begin{aligned}
I_{h c} & =I_{h} \cap\left\{p_{\theta}=c\right\} \\
& =\left\{\left(r, p_{r}\right): p_{r}= \pm \sqrt{2 f_{h}(r)-\frac{c^{2}}{r^{2}}}\right\} \times \mathbb{S}^{1} \\
& \approx g^{-1}(h) \times \mathbb{S}^{1},
\end{aligned}
$$

we can compute the invariant sets $I_{h c}$. Consequently, we can describe the foliation of $I_{h}$ by $I_{h c}$ when $c$ varies.

We note that a point $\left(r^{*}, \theta^{*}, p_{r}^{*}, p_{\theta}^{*}\right)$ is regular for the map $\left(H, p_{\theta}\right)$ if the rank of the matrix

$$
\left.\left(\begin{array}{cccc}
\frac{\partial H}{\partial r} & \frac{\partial H}{\partial \theta} & \frac{\partial H}{\partial p_{r}} & \frac{\partial H}{\partial p_{\theta}} \\
0 & 0 & 0 & 1
\end{array}\right)\right|_{5}
$$


is 2 , and a point which is not regular is critical. A value $(h, c) \in \mathbb{R}^{2}$ is regular if the set $\left(H, p_{\theta}\right)^{-1}(h, c)$ does not contain any critical point, otherwise it is a critical value.

We consider different cases.

Case 1: $K \leq 0$ and $h>0$. In Figure 1(a) if $K<0$ the graphic of $f_{h}(r)$ decreases monotonically from $+\infty$ to $h$, while for $K=0$ the graphic decreases having an inflexion point at $r=7 / B$. It follows from Figure 1(a) that under these assumptions the zero velocity curve is empty and so the Hill region is $R_{h}=\mathbb{R}^{2} \backslash\{(0,0)\}$. Moreover $I_{h}$ is diffeomorphic to $\left(\mathbb{R}^{2} \backslash\{(0,0)\}\right) \times$ $\mathbb{S}^{1}$ because for each point of $R_{h}$ we have a topological circle of velocities. The boundary of $I_{h}$ with $r=0$ corresponds to the collision manifold and the boundary with $r=+\infty$ to the infinity manifold.

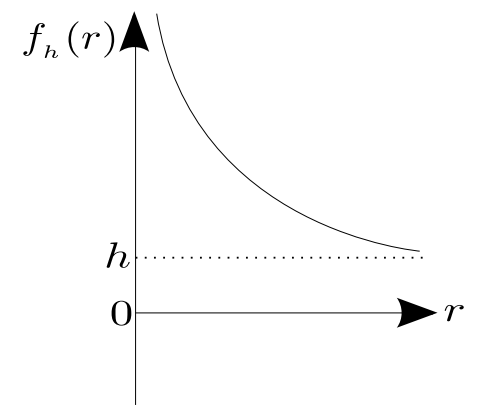

(a)

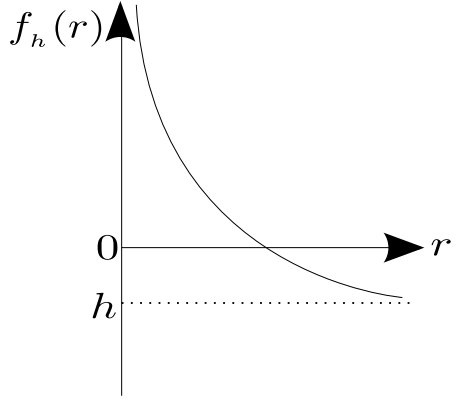

(b)

FiguRE 1. The graph of the function $f_{h}(r)$ : (a) For $K \leq 0$ and $h \geq 0$. (b) For $K \leq 0$ and $h<0$.

Note that $g^{-1}(h)$ is the topological cylinder of Figure 2.

When we consider the curves $\gamma_{h c}=g^{-1}(h) \cap\left\{p_{\theta}=c\right\}$ for each $c \in \mathbb{R}$, we need to distinguish three subcases. There exist two values of $c, \pm c_{1}$, for which the curve $I_{h c}$ is not a manifold, and for these two values the curve $\gamma_{h c}$ is homeomorphic to the shape of the letter $X$. Since $I_{h, \pm c_{1}}$ is not a manifold, then the value $\left(h, \pm c_{1}\right)$ is not regular for the function $\left(H, p_{\theta}\right)$.

If $|c|>c_{1}$, the curve $\gamma_{h c}$ has two components homeomorphic to $\mathbb{R}$, one defined in $0<r \leq r_{1}$ and the other defined in $r_{2} \leq r<\infty$ with $r_{1}<r_{2}$, see Figure 3(a).

If $|c|=c_{1}$, the curve $\gamma_{h c}$ has only one component topologically homeomorphic to the shape of the letter $X$, which can be obtained as a limiting case of Figure 2 when $|c| \rightarrow c_{1}$ and $r_{1}$ and $r_{2}$ tend to the same value, see Figure 3(b). 


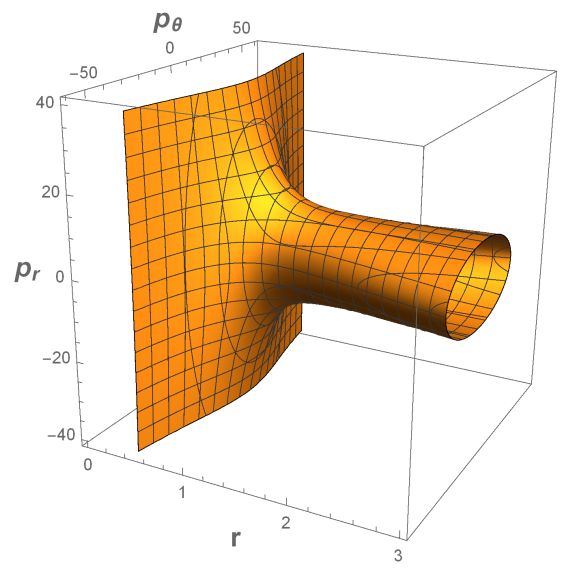

Figure 2. The surface $g^{-1}(h)$ for $K \leq 0$ and $h>0$.

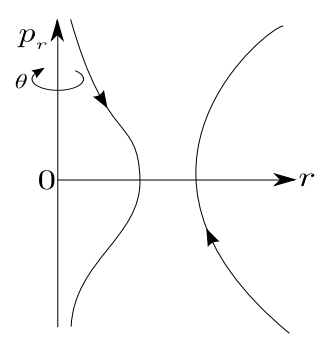

(a)

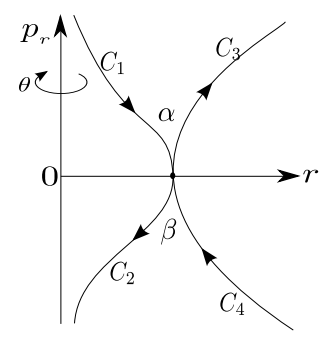

(b)

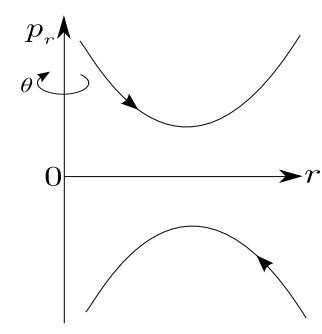

(c)

Figure 3. Graphs of the curves $\gamma_{h c}$ when $K \leq 0$ and $h \geq 0$ :

(a) For $|c|>c_{1}$. (b) For $|c|=c_{1}$. (c) For $|c|<c_{1}$.

If $|c|<c_{1}$, the curve $\gamma_{h c}$ has two components, each one defined for all $r>0$ and homeomorphic to $\mathbb{R}$, see Figure 3(c).

Clearly the manifold $I_{h}$ is diffeomorphic to a solid torus of dimension three without the boundary and without the central circular axis. Using topology techniques we have that a solid torus of dimension three without the boundary and without the central circular axis is homeomorphic to $\mathbb{S}^{3} \backslash$ $\left\{\mathbb{S}^{1} \cup \mathbb{S}^{1}\right\}$. We claim that the foliation of $I_{h}$ by the subsets $I_{h c}$ varying $c$ can be obtained by rotating Figure 4 around the $u$ axis. Now we prove the claim.

Assume that $|c|>c_{1}$. From Figure 3(a) the manifold $I_{h c}$ is formed by two cylinders. In the cylinder for which $r \in\left(0, r_{1}\right]$ the orbits start in ejection and end in collision. On the cylinder for which $r \in\left[r_{2}, \infty\right)$ the orbits start and end at infinity hyperbolically, i.e. with radial velocity $|\dot{r}| \neq 0$. 
Assume that $c=c_{1}$ (respectively, $c=-c_{1}$ ). From Figure 3(a) the invariant set $I_{h c}$ is formed by a periodic orbit $\alpha$ (respectively, $\beta$ ) and four cylinders $C_{i}, i=1,2,3,4$, having a common boundary formed by the periodic orbit $\alpha$ (respectively, $\beta$ ). The cylinders $C_{1}$ and $C_{4}$ are the stable manifold of $\alpha$ (respectively, $\beta$ ), and the cylinders $C_{2}$ and $C_{3}$ are the unstable manifold of $\alpha$ (respectively, $\beta$ ). On the cylinder $C_{1}$ the orbits start at ejection and end in $\alpha$ (respectively, $\beta$ ), on the cylinders $C_{2}$ the orbits start in $\alpha$ (respectively, $\beta$ ) and ends in collision, on the cylinder $C_{3}$ the orbits start in $\alpha$ (respectively, $\beta$ ) and end at infinity hyperbolically, and finally the orbits on the cylinder $C_{4}$ start at infinity hyperbolically and end in $\alpha$ (respectively, $\beta$ ).

Assume $|c|<c_{1}$. From Figure 3(c) the manifold $I_{h c}$ is formed by two cylinders. On one of the cylinders the orbits start in ejection and end at infinity hyperbolically and on the other cylinder the orbits start at infinity hyperbolically and end in collision.

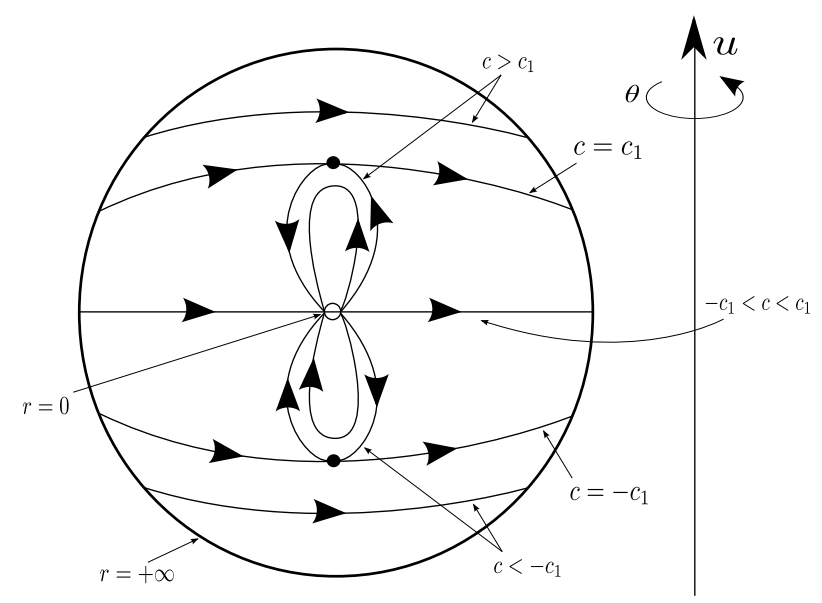

Figure 4. Manifold $I_{h} / S^{1}$ for $K \leq 0$ and $h>0$.

Case 2: $K \leq 0$ and $h=0$. It follows from Figure 1(b) that the Hill region is again $R_{h}=\mathbb{R}^{2} \backslash\{(0,0)\}$, and so $I_{h}$ is homeomorphic to $\mathbb{S}^{3} \backslash\left\{\mathbb{S}^{1} \cup \mathbb{S}^{1}\right\}$ as in Case 1. In Figure 5 we have the surface $g^{-1}(h)$ for $K \leq 0$ and $h=0$.

If $c \in \mathbb{R} \backslash\{0\}$ the curve $\gamma_{h c}$ has one component homeomorphic to $\mathbb{R}$, see Figure 6(a).

If $c=0$ the curve $\gamma_{h c}$ has two components homeomorphic to $\mathbb{R}$, see Figure 6(b).

We claim that the foliation of $I_{h}$ by the subsets $I_{h c}$ varying $c$ can be obtained by rotating Figure 7 around the $u$ axis. Now we prove the claim. 


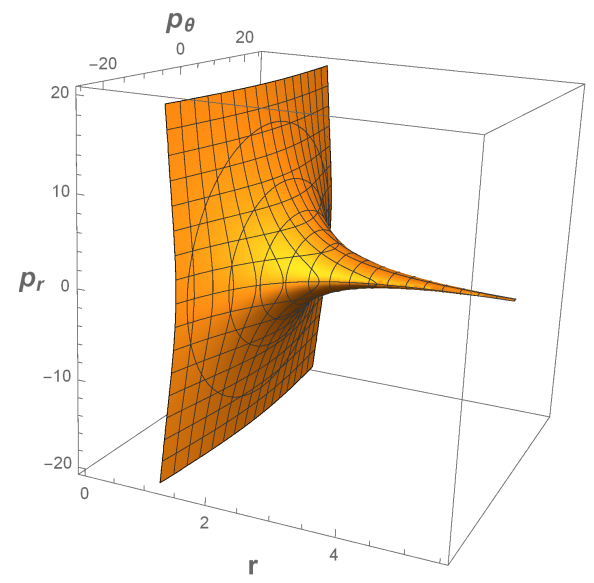

Figure 5. The surface $g^{-1}(h)$ for $K \leq 0$ and $h=0$.

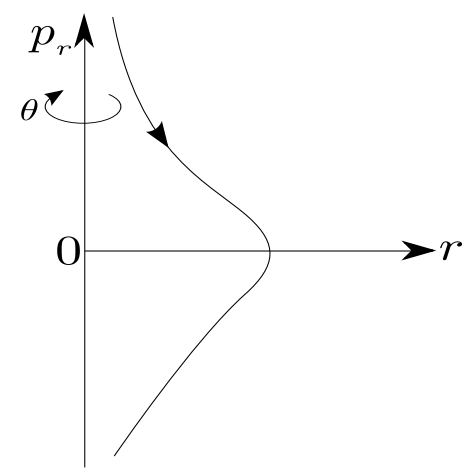

(a)

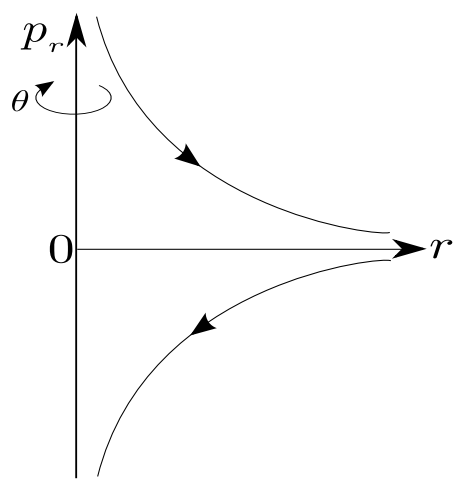

(b)

Figure 6 . Graphs of the curves $\gamma_{h c}$ when $K \leq 0$ and $h=0$ :

(a) For $c \in \mathbb{R} \backslash\{0\}$. (b) For $c=0$.

Assume that $c \in \mathbb{R} \backslash\{0\}$. From Figure 8 we have that the manifold $I_{h c}$ is formed by one cylinder. In this cylinder the orbits start in ejection and end in collision.

Assume that $c=0$. From Figure 6(b) the invariant set $I_{h c}$ is formed by two cylinders. The orbits in the cylinder $p_{r}>0$ start in ejection and end at infinity parabolically, i.e. they reach the infinity with zero radial velocity. In the cylinder $p_{r}<0$ the orbits start at infinity parabolically and end at collision. 


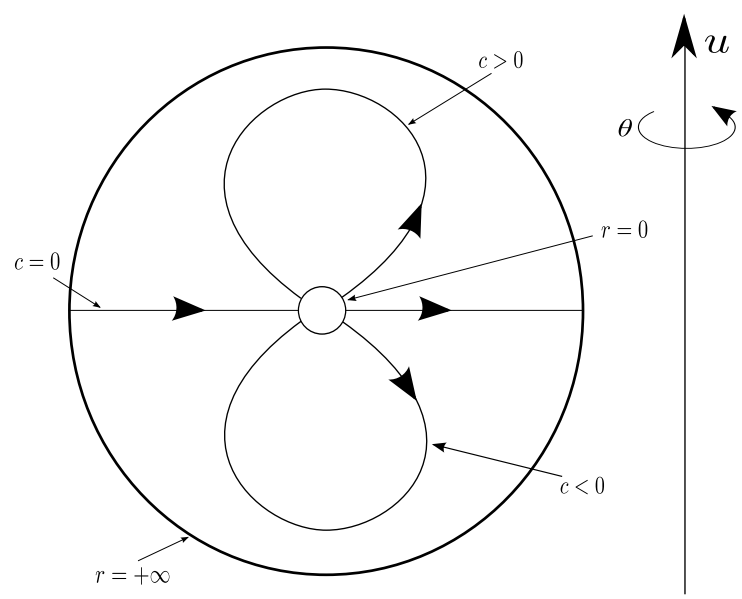

Figure 7. Manifold $I_{h} / S^{1}$ for $K \leq 0$ and $h=0$.

Case 3: $K \leq 0$ and $h<0$. It follows from Figure 1(b) that the zero velocity curve is a circle centered at the origin of coordinates. Then the Hill region $\pi\left(I_{h}\right)$ is a punctured closed disc centered at the origin whose boundary is the zero velocity curve, that is, $R_{h}$ is diffeomorphic to $(0, a] \times \mathbb{S}^{1}$ where $a>0$. So $I_{h}$ is diffeomorphic to an open solid torus whose boundary corresponds to the collision manifold, the central axis of this solid torus is the zero velocity curve. Indeed, $R_{h} \approx\left\{(r, \theta) \in(0, a] \times \mathbb{S}^{1}\right\}$. The circle $r=a$ is the zero velocity curve. So for each ray $(0, a] \times\left\{\theta=\theta_{0}\right\}$ we have a circle of velocities for each point $\left(r, \theta_{0}\right)$ with $r \in(0, a)$. The radius of this circle varies continuously with $r$ and becomes zero at $r=a$. Taking into account all these circles together and varying $\theta_{0} \in \mathbb{S}^{1}$ we obtain the solid torus without the boundary with a "central axis" formed by the zero velocity circle, and the boundary of this solid torus corresponds to the collision manifold. Applying topology arguments we have that a solid torus without the boundary is homeomorphic to $\mathbb{S}^{3} \backslash \mathbb{S}^{1}$, so $I_{h} \approx \mathbb{S}^{3} \backslash \mathbb{S}^{1}$.

On the other hand the surface $g^{-1}(h)$ is the topological plane of Figure 8.

The curves $\gamma_{h c}=g^{-1}(h) \cap\left\{p_{\theta}=c\right\}$ for each $c \in \mathbb{R}$ are defined for all $r$ in $0<r<r(c)$ and are homeomorphic to $\mathbb{R}$. The manifold $I_{h}$ can be obtained by rotating Figure 9 around the $u$ axis. In this picture we can see one cylinder $I_{h c}$ for every $c \in \mathbb{R}$ foliating $I_{h}$. The orbits on this cylinder start in ejection and end in collision. We note that only the orbits on the cylinder $I_{h 0}$ pass through the zero velocity curve.

Case 4: $K>0$ and $2 h<f_{1}<f_{2}$. We consider different subcases, see Figure 10(a).

Subcase 4.1: $f_{1}>0$. The dynamics of this case with $h>0$ is the same as the one of Case 1 and with $h=0$ is the same as the one of Case 2, while 


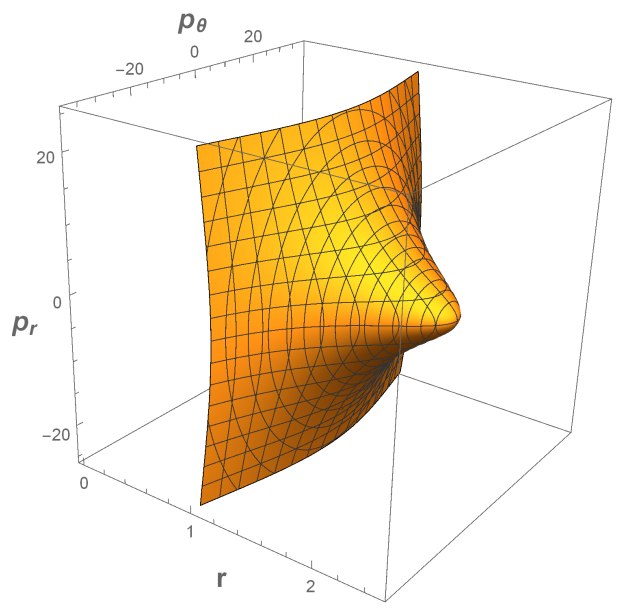

Figure 8. The surface $g^{-1}(h)$ for $K \leq 0$ and $h<0$.

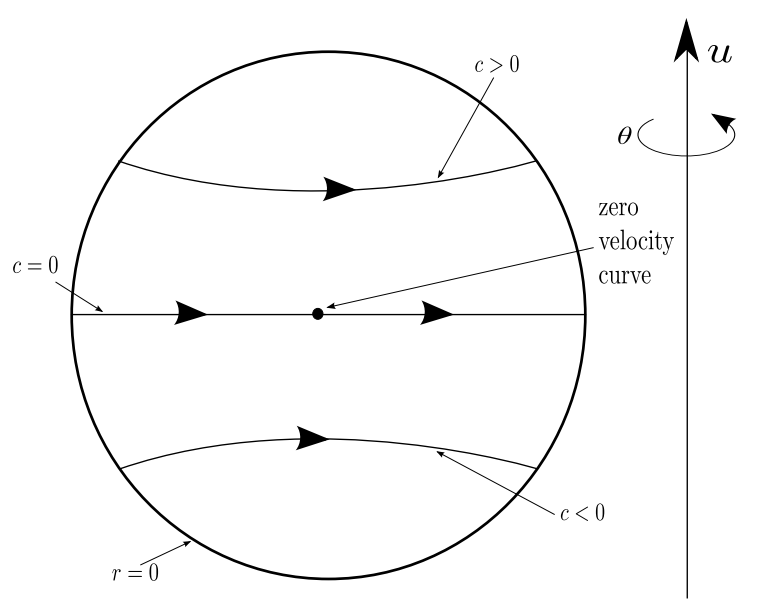

Figure 9. Manifold $I_{h} / S^{1}$ for $K \leq 0$ and $h<0$.

the dynamics in this case with $h<0<f_{1}$ is dynamically the same as the one of Case 3.

Subcase 4.2: $f_{1}=0$. From Figure 10(a) we see that the zero velocity curve is formed by two circles centered at the origin of coordinates. The Hill region is formed by a closed disc of radius $d$ centered at the origin without the origin, the external boundary of this disc is the biggest circle $r=d$ of the zero velocity curve and additionally there is a circle $r=a<d$ of the zero velocity curve contained in the interior of this disc. 


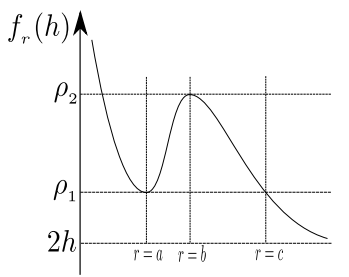

(a)

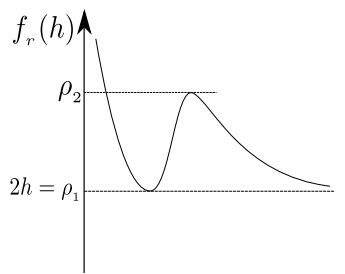

(b)

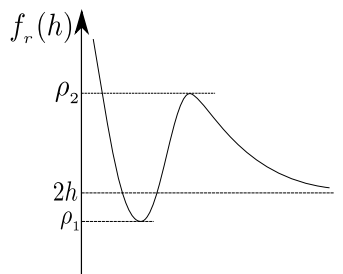

(c)

FiguRE 10. Graph of the function $f_{h}(r)$ when $K>0$ : (a) For $2 h<f_{1}$. (b) For $2 h=f_{1}$. (c) For $2 h>f_{1}$.

The part of the Hill region $\left\{(r, \theta): r \in(0, a], \theta \in \mathbb{S}^{1}\right\}$ corresponds to the projection of an open solid torus contained in $I_{h}$ whose boundary is the collision manifold. This open solid torus is similar to the one of Case 3. We claim that the part of the Hill region $\left\{(r, \theta): r \in[a, d], \theta \in \mathbb{S}^{1}\right\}$ corresponds to the projection of a set homeomorphic to $\mathbb{S}^{2} \times \mathbb{S}^{1}$ contained in $I_{h}$. Indeed, for each $\theta_{0} \in \mathbb{S}^{1}$ and for each point of the segment $\left\{\left(r, \theta_{0}\right): r \in(a, d)\right\}$ we have a circle of velocity which reduces to a point at $r=a$ and $r=d$. So a sphere $\mathbb{S}^{2}$ of velocities of $I_{h}$ projects onto the segment $\left\{\left(r, \theta_{0}\right): r \in[a, d]\right\}$ for all $\theta_{0} \in \mathbb{S}^{1}$. Hence the claim is proved.

In short, $I_{h}$ is formed by the union of an open solid torus and $\mathbb{S}^{2} \times \mathbb{S}^{1}$ but the central axis of the solid torus formed by the circle of zero velocity $r=a$ is identified with a circle of $\mathbb{S}^{2} \times \mathbb{S}^{1}$.

The set $I_{h}$ also can be obtained rotating Figure 11 with respect to the $u$-axis. In Figure 11 we have drawn the surface $g^{-1}(h)$ for $K>0$ and $h=0$.

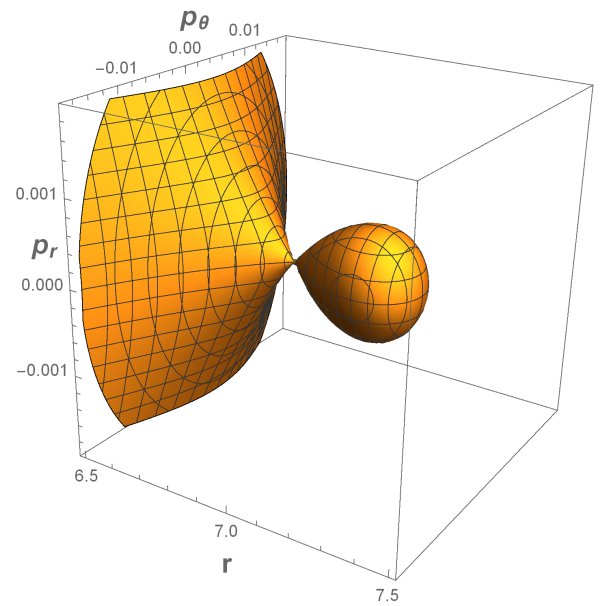

Figure 11. The surface $g^{-1}(h)$ for $K>0$ and $0=f_{1}<2 h$. 
The invariant sets $I_{h c}$ in this case are not manifolds of dimension two at three values of $c$, namely $-c_{1}, 0$ and $c_{1}$. Consequently the values $\left(h, \pm c_{1}\right)$ and $(h, 0)$ are not regular for the function $\left(H, p_{\theta}\right)$.

If $|c|>c_{1}$ from Figure 12(a) we have that $I_{h c}$ is a cylinder. The orbits on this cyclinder are of ejection-collision.

If $|c|=c_{1}$ from Figure 12(b) we obtain that $I_{h c}$ is the union of a cylinder with a periodic orbit. Again the orbits of the cylinder are of ejectioncollision.

If $0<|c|<c_{1}$ from Figure 12(c) we get that $I_{h c}$ is the union of a cylinder with a torus. Again the orbits on the cylinder are of ejection-collision. The orbits on the torus can be either quasiperiodic and consequently dense in the torus, or periodic.

If $c=0$ from Figure $12(\mathrm{~d})$ we have that $I_{h c}$ has only one component formed by three cylinders which share the circle of singular points $r=a$. The cylinder $C_{1}$ is formed by orbits of ejection which end in the circle $r=a$. The orbits on the cylinder $C_{3}$ start at the circle $r=a$ and end at collision, and the orbits on the cylinder $C_{2}$ start and end at $r=a$.

In summary the foliation of $I_{h}$ but the sets $I_{h c}$ varying $c$ can be obtained by rotating Figure 13(a) around the $u$ axis, with the union of $\mathbb{S}^{2} \times \mathbb{S}^{1}$ of Figure 13(b), and identifying the zero velocity circle $r=a$ contained in both figures. In Figure 13(b) appears the foliation of $\mathbb{S}^{2} \times \mathbb{S}^{1}$ by the invariant sets $I_{h c}$. This foliation corresponds to the Hopf foliation of $\mathbb{S}^{3} \approx \mathbb{S}^{2} \times \mathbb{S}^{1}$. We must identify the points (of the two surfaces of the cones glued by their bases) which are symmetric with respect to the plane containing the common bases.

Subcase 4.3: $f_{1}<0<f_{2}$. It follows from Figure 10(a) that the zero velocity curve is formed by three circles centered at the origin of coordinates. The Hill region $\pi\left(I_{h}\right)$ has two connected components, one is formed by a closed disc centered at the origin without the origin, the external boundary of this disc is the smallest circle of the zero velocity curve, and the other component is a closed crown whose two boundaries are the other two circles of the zero velocity curve. The manifold $I_{h}$ has two connected components, one is homeomorphic to a solid torus having its central axis formed by the smallest circle of the zero velocity curve and its boundary corresponds to the collision manifold (see for more details Case 3), and the other component is homeomorphic to $\mathbb{S}^{2} \times \mathbb{S}^{1}$ containing the two biggest circles of the zero velocity curve (see for more details Subcase 4.2). The set $I_{h}$ which is homeomorphic to the union of an open solid torus with $\mathbb{S}^{2} \times \mathbb{S}^{1}$ can also be obtained rotating Figure 14(a) with respect to the $u$-axis and adding to it the Figure 14(b). In this last figure it is presented the foliation of the sphere $\mathbb{S}^{3}$ identifying the points (of the surfaces of the cones glued by their bases) which are symmetric with respect to the plane containing the 


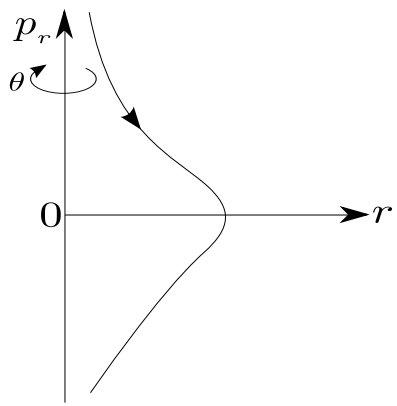

(a)

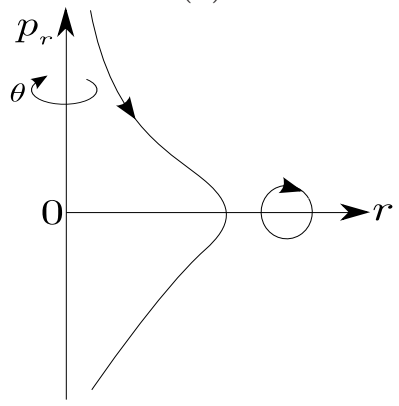

(c)

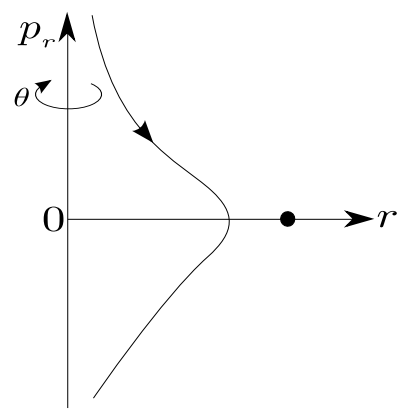

(b)

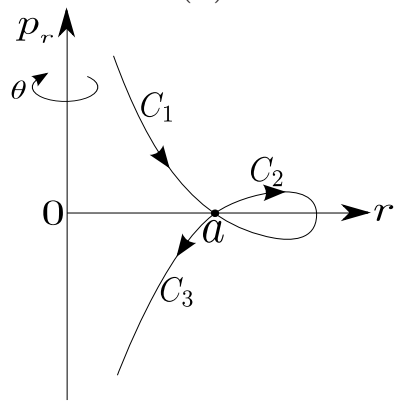

(d)

Figure 12. Graph of the curves $\gamma_{h c}$ when $K>0$ and $2 h<$ $f_{1}=0$ : (a) For $|c|>c_{1}$. (b) For $|c|=c_{1}$. (c) For $0<|c|<c_{1}$. (d) For $c=0$.

common bases. In Figure 14(b) we also can see how the flow moves on the tori $I_{h c}$. In Figure 14 we have drawn the surface $g^{-1}(h)$ for $K>0$ and $f_{1}<0<f_{2}$.

There exist two values of $c, \pm c_{1} \neq 0$ for which the set $I_{h c}$ is not a manifold of dimension two, so the values $\left(h, \pm c_{1}\right)$ are not regular for the function $\left(H, p_{\theta}\right)$.

If $|c|>c_{1}$, from Figure 15(a) we have that $I_{h c}$ is a cylinder whose orbits are of ejection-collision.

If $|c|=c_{1}$ from Figure 12(b) we obtain that $I_{h c}$ is the union of a cylinder with a periodic orbit and the orbits in the cylinder are of ejection-collision.

Finally, if $|c|<c_{1}$, from Figure 15(c) we have that $I_{h c}$ is the union of a cylinder with a torus. Again the orbits on the cylinder are of ejectioncollision, and the orbits on the torus can be either quasiperiodic, or periodic. 


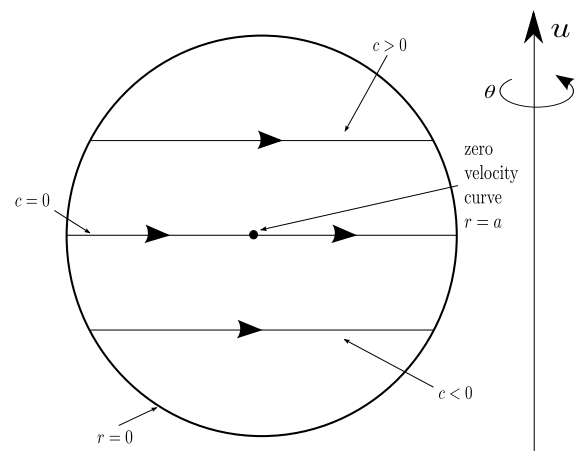

(a)

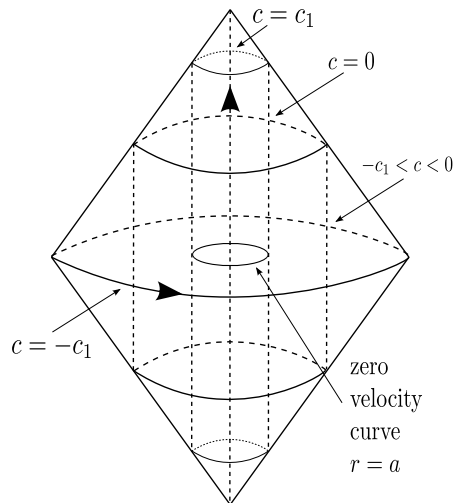

(b)

Figure 13. Manifold $I_{h} / S^{1}$ for $K>0$ and $f_{1}=0>2 h$.

This manifold has two components (a) and (b).

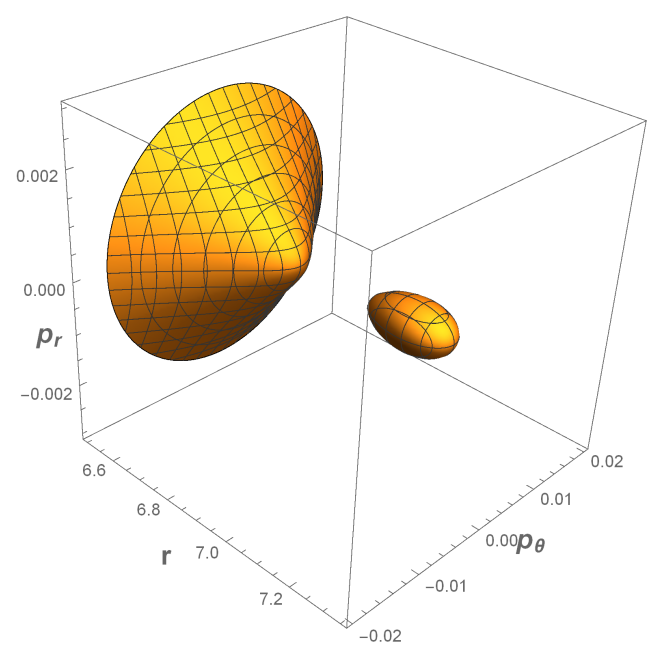

Figure 14. The surface $g^{-1}(h)$ for $K>0$ and $h<f_{1}<0<f_{2}$.

In short, the foliation of $I_{h}$ by the sets $I_{h c}$ varying $c$ can be obtained by rotating Figure 13(a) around the $u$-axis, with the union of $\mathbb{S}^{2} \times \mathbb{S}^{1}$ of Figure 13(b).

Subcase 4.4: $f_{2}=0$. It follows from Figure 10(a) that the zero velocity curve is formed by two circles centered at the origin of coordinates. The Hill region $\pi\left(I_{h}\right)$ has two connected components, one is formed by a closed disc centered at the origin without the origin, the external boundary of this disc 
is the smallest circle of the zero velocity curve, and the other component is the biggest circle of the zero velocity curve. So $I_{h}$ has two connected components, one homeomorphic to a solid torus having as central axis the smallest circle of the zero velocity curve. whose boundary corresponds to the collision manifold (see Case 3), and the other is the biggest circle of the zero velocity curve, i.e. a circle of equilibria. Here the surface $g^{-1}(h)$ is the one given in Figure 8 with an additional isolated point. The foliation of $I_{h}$ by the invariant sets $I_{h c}$ is a cylinder if $c \in \mathbb{R} \backslash\{0\}$, and the union of cylinder with a circle of equilibria if $c=0$.

Subcase 4.5: $f_{2}<0$. The dynamics of this case is the same as the one of Case 3.

Case 5: If $K>0$ and $f_{1}=2 h<f_{2}$. We consider different subcases, see Figure 10(b).

Subcase 5.1: $0<f_{1}$, or $f_{1}<0<f_{2}$, or $f_{2}=0$, or $f_{2}<0$. The dynamics of case $0<f_{1}$ is the same as the one of Case 1 . The dynamics of case $f_{1}<0<f_{2}$ is the same as the one of Subcase 4.3. The dynamics of case $f_{2}=0$ is the same as the one of Subcase 4.4, and the dynamics of case $f_{2}<0$ is the same as the one of Case 3 .

Subcase 5.2: $f_{1}=2 h=0$. It follows from Figure 10(b) that the zero velocity curve is formed by a circle centered at the origin of coordinates, the Hill region $\pi\left(I_{0}\right)$ is $\mathbb{R}^{2} \backslash\{(0,0)\}$, but it contains in its interior the zero velocity curve, and $I_{h}$ is homeomorphic to two solid tori without the boundary which have identified their "central axis" with the zero velocity curve, the boundary of one of the solid tori is the collision manifold and the boundary of the other solid tori is the infinity manifold.

From Figure 15 the surface $g^{-1}(0)$ topologically is formed by two planes with a common point identified. The curves $\gamma_{0 c}=g^{-1}(0) \cap\left\{p_{\theta}=c\right\}$ for $c \in \mathbb{R} \backslash\{0\}$ are topologically the ones of Figure 3(a), and for $c=0$ are topologically the ones of Figure 3(b). The foliation of $I_{h}$ by the invariant sets $I_{h c}$ varying $c$ can be obtained by rotating Figure 9 with an additional circle $\mathbb{S}^{1}$ of equilibria.

If $c \in \mathbb{R} \backslash\{0\}$ then $I_{0 c}$ is formed by two cylinders. In one cylinder the orbits are of ejection-collision, and in the other cylinder the orbits start and end at infinity parabolically.

If $c=0$ the topology of $I_{00}$ and the dynamics on it is the same as for the sets $I_{h, \pm c_{1}}$ of Case 1, with the difference that the periodic orbit $\alpha$ in $I_{h, \pm c_{1}}$ now becomes a circle of equilibria.

The foliation of $I_{0}$ by the sets $I_{0 c}$ varying $c$ can be obtained by rotating Figure 16 around the $u$-axis. After the rotation with respect to the $u$-axis 


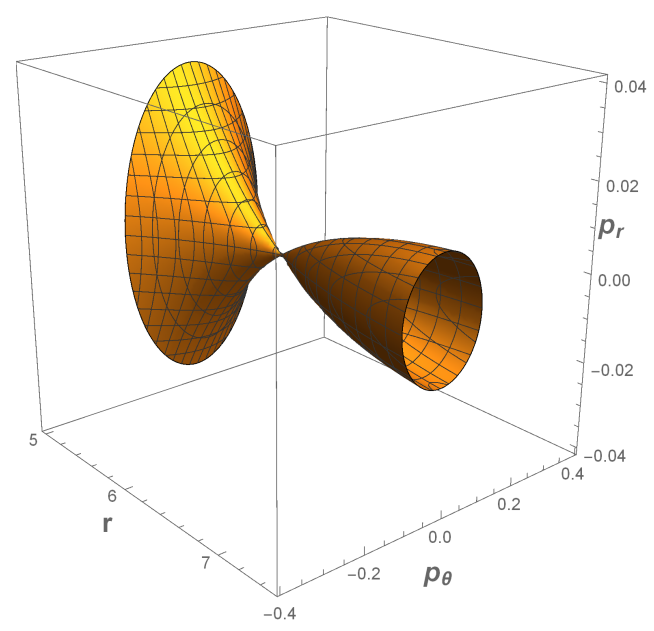

Figure 15. The surface $g^{-1}(h)$ for $K>0$ and $2 h=f_{1}=0$.

\begin{tabular}{|c|c|c|c|c|}
\hline & $I_{h}$ & $g^{-1}(h)$ & $I_{h c}$ & $I_{h} / \mathbb{S}^{I}$ \\
\hline$h>0$ & $\mathbb{S}^{3} \backslash\left\{\mathbb{S}^{1} \cup \mathbb{S}^{1}\right\}$ & 2 & 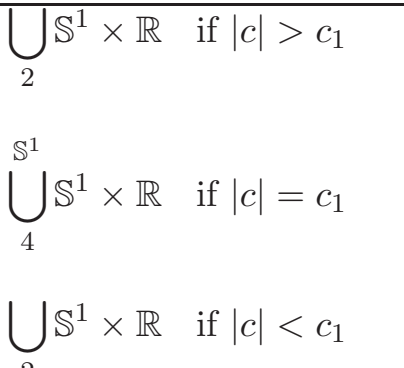 & 4 \\
\hline$h=0$ & $\mathbb{S}^{3} \backslash\left\{\mathbb{S}^{1} \cup \mathbb{S}^{1}\right\}$ & 5 & $\begin{array}{ll}\mathbb{S}^{1} \times \mathbb{R} & \text { if } c \in \mathbb{R} \backslash\{0\} \\
\bigcup \mathbb{S}^{1} \times \mathbb{R} & \text { if } c=0\end{array}$ & 7 \\
\hline$h<0$ & $\mathbb{S}^{3} \backslash \mathbb{S}^{1}$ & 8 & $\mathbb{S}^{1} \times \mathbb{R} \quad$ if $c \in \mathbb{R}$ & 9 \\
\hline
\end{tabular}

TABLE 1 . The invariant set $I_{h}$ and its foliation by $I_{h c}$ for $K \leq 0$. In the column $g^{-1}(h)$ we indicate the number of the figure where the surface $g^{-1}(h)$ is drawn, and in the column $I_{h} / \mathbb{S}^{1}$ we indicate the number of the figure where it is described the foliation of $I_{h}$ by the invariant sets $I_{h c}$.

the two central axes of both open solid tori in the zero velocity curve must be identified. 


\begin{tabular}{|c|c|c|c|c|c|}
\hline & $I_{h}$ & $g^{-1}(h)$ & $I_{h c}$ & & $I_{h} / \mathbb{S}^{1}$ \\
\hline$f_{1}>0, h>0$ & $\mathbb{S}^{3} \backslash\left\{\mathbb{S}^{1} \cup \mathbb{S}^{1}\right\}$ & 2 & $\begin{array}{l}\bigcup_{2} \mathbb{S}^{1} \times \mathbb{R} \\
\bigcup_{4}^{\mathbb{S}^{1}} \mathbb{S}^{1} \times \mathbb{R} \\
\bigcup \mathbb{S}^{1} \times \mathbb{R}\end{array}$ & $\begin{array}{l}\text { if }|c|>c_{1} \\
\text { if }|c|=c_{1} \\
\text { if }|c|<c_{1}\end{array}$ & 4 \\
\hline$h=0$ & $\mathbb{S}^{3} \backslash\left\{\mathbb{S}^{1} \cup \mathbb{S}^{1}\right\}$ & 5 & $\begin{array}{l}\mathbb{S}^{1} \times \mathbb{R} \\
\bigcup \mathbb{S}^{1} \times \mathbb{R}\end{array}$ & $\begin{array}{l}\text { if } c \in \mathbb{R} \backslash\{0\} \\
\text { if } c=0\end{array}$ & 7 \\
\hline$h<0<f_{1}$ & $\mathbb{S}^{3} \backslash \mathbb{S}^{1}$ & 8 & $\mathbb{S}^{1} \times \mathbb{R}$ & if $c \in \mathbb{R}$ & 9 \\
\hline$f_{1}=0$ & $\left(\mathbb{S}^{3} \backslash \mathbb{S}^{1}\right) \bigcup^{\mathbb{S}^{1}} \mathbb{S}^{2} \times \mathbb{S}^{1}$ & 11 & $\begin{array}{c}\mathbb{S}^{1} \times \mathbb{R} \\
\mathbb{S}^{1} \bigcup \mathbb{S}^{1} \times \mathbb{R} \\
\mathbb{S}^{1} \times \mathbb{R} \bigcup \mathbb{S}^{1} \times \mathbb{S}^{1} \\
\mathbb{S}^{1} \mathbb{S}^{1} \times \mathbb{R}\end{array}$ & $\begin{array}{l}\text { if }|c|>c_{1} \\
\text { if }|c|=c_{1} \\
\text { if } 0<|c|<c_{1} \\
\text { if } c=0\end{array}$ & 13 \\
\hline$f_{1}<0<f_{2}$ & $\left(\mathbb{S}^{3} \backslash \mathbb{S}^{1}\right) \bigcup \mathbb{S}^{2} \times \mathbb{S}^{1}$ & 14 & $\begin{array}{c}\mathbb{S}^{1} \times \mathbb{R} \\
\mathbb{S}^{1} \bigcup \mathbb{S}^{1} \times \mathbb{R} \\
\mathbb{S}^{1} \times \mathbb{R} \bigcup \mathbb{S}^{1} \times \mathbb{S}^{1}\end{array}$ & $\begin{array}{l}\text { if }|c|>c_{1} \\
\text { if }|c|=c_{1} \\
\text { if }|c|<c_{1}\end{array}$ & 13 \\
\hline$f_{2}=0$ & $\left(\mathbb{S}^{3} \backslash \mathbb{S}^{1}\right) \bigcup \mathbb{S}^{1}$ & $8^{*}$ & $\begin{array}{c}\mathbb{S}^{1} \times \mathbb{R} \\
\mathbb{S}^{1} \times \mathbb{R} \bigcup \mathbb{S}^{1}\end{array}$ & $\begin{array}{l}\text { if } c \in \mathbb{R} \backslash\{0\} \\
\text { if } c=0\end{array}$ & $9^{*}$ \\
\hline$f_{2}<0$ & $\mathbb{S}^{3} \backslash \mathbb{S}^{1}$ & 8 & $\mathbb{S}^{1} \times \mathbb{R}$ & if $c \in \mathbb{R}$ & 9 \\
\hline
\end{tabular}

TABle 2. The invariant set $I_{h}$ and its foliation by $I_{h c}$ for $K>0$ and $2 h<f_{1}<f_{2}$. The $8^{*}$ in the column $g^{-1}(h)$ indicates that the surface $g^{-1}(h)$ is the one of Figure 8 with an additional isolated point. The $9^{*}$ in the column of $I_{h} / \mathbb{S}^{1}$ indicates that the foliation of $I_{h}$ by the invariant sets $I_{h c}$ is the same as the one in Figure 9 with an additional circle $\mathbb{S}^{1}$ of equilibria. 

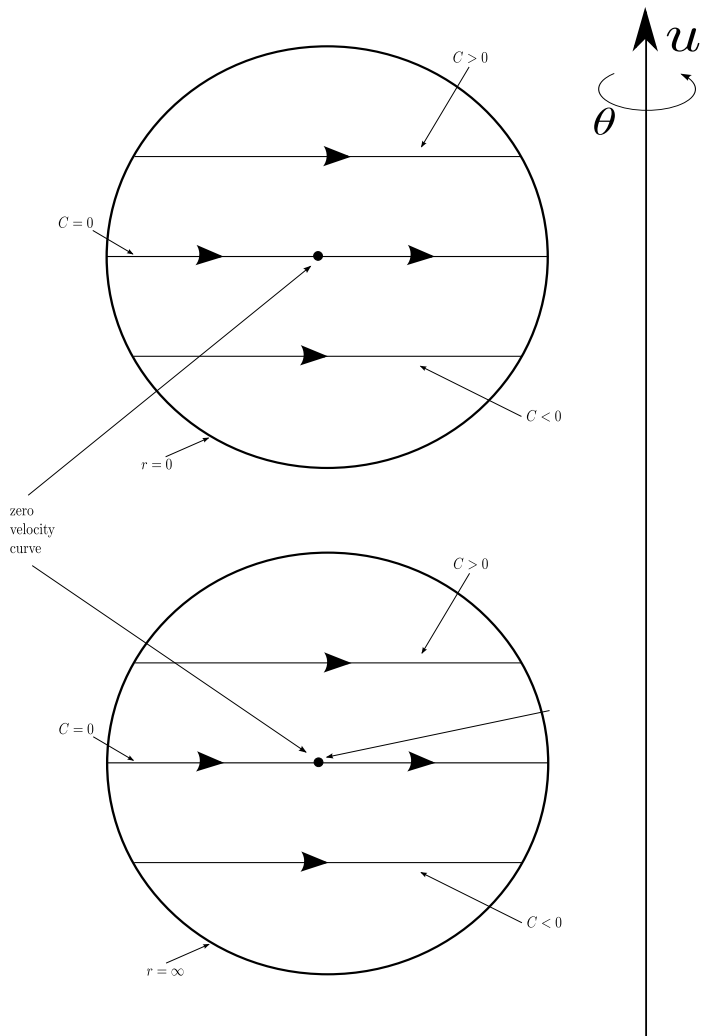

FiguRE 16. Manifold $I_{h} / S^{1}$ for $K>0$ and $f_{1}=0=2 h<f_{2}$.

Case 6 : If $K>0$ and $f_{1}<2 h<f_{2}$. We consider different subcases, see Figure 10(c)

Subcase 6.1: $0<f_{1}$, or $f_{1}=0$, or $2 h<0<f_{2}$, or $f_{2}=0$, or $f_{2}<0$. The dynamics in the case $0<f_{1}$ is the same as the one of Case 1 . The dynamics in the case $f_{1}=0$ is the same as the one of Subcase 5.2 but the orbits starting and ending at infinity are now hyperbolic instead of parabolic. The dynamics in the case $2 h<0<f_{2}$ is the same as the one of Subcase 4.3. Moreover, the dynamics in the case $f_{2}=0$ is the same as the one of Subcase 4.4, and finally the dynamics of the last case $f_{2}<0$ is the same as the one of Case 3.

Subcase 6.2: $f_{1}<0 \leq 2 h$. It follows from Figure 13(c) that the zero velocity curve is formed by two circles centered at the origin. TheHill region $\pi\left(I_{h}\right)$ is formed by two topological discs, one is a closed disc centered at the origin without the origin whose external boundary is the smallest circle of the zero velocity curve, the other topological disc is of the form $\left[r_{1}, \infty\right) \times \mathbb{S}^{1}$ and the circle $r=r_{1}$ is the biggest circle of the zero velocity curve. 


\begin{tabular}{|c|c|c|c|c|c|}
\hline & $I_{h}$ & $g^{-1}(h)$ & \multicolumn{2}{|c|}{$I_{h c}$} & $I_{h} / \mathbb{S}^{1}$ \\
\hline$f_{1}>0$ & $\mathbb{S}^{3} \backslash\left\{\mathbb{S}^{1} \cup \mathbb{S}^{1}\right\}$ & 2 & $\begin{array}{l}\bigcup_{2} \mathbb{S}^{1} \times \mathbb{R} \\
\bigcup_{4}^{\mathbb{S}^{1}} \mathbb{S}^{1} \times \mathbb{R} \\
\bigcup \mathbb{S}^{1} \times \mathbb{R}\end{array}$ & $\begin{array}{l}\text { if }|c|>c_{1} \\
\text { if }|c|=c_{1} \\
\text { if }|c|<c_{1}\end{array}$ & 4 \\
\hline$f_{1}=2 h=0$ & $\bigcup_{2}^{\mathbb{S}^{1}}\left(\mathbb{S}^{3} \backslash \mathbb{S}^{1}\right)$ & 15 & $\begin{array}{l}\bigcup_{2}^{2} \mathbb{S}^{1} \times \mathbb{R} \\
\bigcup^{1} \mathbb{S}^{1} \times \mathbb{R}\end{array}$ & $\begin{array}{l}\text { if } c \in \mathbb{R} \backslash\{0\} \\
\text { if } c=0\end{array}$ & 16 \\
\hline$f_{1}<0<f_{2}$ & $\left(\mathbb{S}^{3} \backslash \mathbb{S}^{1}\right) \bigcup \mathbb{S}^{2} \times \mathbb{S}^{1}$ & 14 & $\begin{array}{c}\mathbb{S}^{1} \times \mathbb{R} \\
\mathbb{S}^{1} \bigcup \mathbb{S}^{1} \times \mathbb{R} \\
\mathbb{S}^{1} \times \mathbb{R} \bigcup \mathbb{S}^{1} \times \mathbb{S}^{1}\end{array}$ & $\begin{array}{l}\text { if }|c|>c_{1} \\
\text { if }|c|=c_{1} \\
\text { if }|c|<c_{1}\end{array}$ & 13 \\
\hline$f_{2}=0$ & $\left(\mathbb{S}^{3} \backslash \mathbb{S}^{1}\right) \bigcup \mathbb{S}^{1}$ & $8^{*}$ & $\begin{array}{c}\mathbb{S}^{1} \times \mathbb{R} \\
\mathbb{S}^{1} \times \mathbb{R} \bigcup \mathbb{S}^{1}\end{array}$ & $\begin{array}{l}\text { if } c \in \mathbb{R} \backslash\{0\} \\
\text { if } c=0\end{array}$ & $9^{*}$ \\
\hline$f_{2}<0$ & $\mathbb{S}^{3} \backslash \mathbb{S}^{1}$ & 8 & $\mathbb{S}^{1} \times \mathbb{R}$ & if $c \in \mathbb{R}$ & 9 \\
\hline
\end{tabular}

TABLE 3 . The invariant set $I_{h}$ and its foliation by $I_{h c}$ for $K>0$ and $f_{1}=2 h<f_{2}$.

The manifold $I_{h}$ is homeomorphic to the union of two open solid tori, each solid torus has its central axis formed by one of the circles of the zero velocity curve, and the boundary of the solid tori having as central axis the smallest circle of the zero velocity is the collision manifold and the boundary of the other solid torus is the infinity manifold. Hence, $I_{h}$ is diffeomorphic to $\left(\mathbb{S}^{3} \backslash \mathbb{S}^{1}\right) \cup\left(\mathbb{S}^{3} \backslash \mathbb{S}^{1}\right)$. The surface $g^{-1}(h)$ is the one of Figure 15 but now the two surfaces of that figure do not share a common point.

The foliation of $I_{h}$ by the sets $I_{h c}$ and the dynamics on each $I_{h c}$ is described in Figure 16 without identifying the two central axes of both tori, because now the zero velocity curve is formed by two distinct circles.

In tables 1-4, we summarize the foliation of $I_{h}$ by $I_{h c}$ for all the values of $K$ and $h$. 


\begin{tabular}{|c|c|c|c|c|c|}
\hline & $I_{h}$ & $g^{-1}(h)$ & \multicolumn{2}{|c|}{$I_{h c}$} & $I_{h} / \mathbb{S}^{1}$ \\
\hline$f_{1}>0$ & $\mathbb{S}^{3} \backslash\left\{\mathbb{S}^{1} \cup \mathbb{S}^{1}\right\}$ & 2 & $\begin{array}{l}\bigcup_{2} \mathbb{S}^{1} \times \mathbb{R} \\
\bigcup_{4}^{\mathbb{S}^{1}} \mathbb{S}^{1} \times \mathbb{R} \\
\bigcup \mathbb{S}^{1} \times \mathbb{R}\end{array}$ & $\begin{array}{l}\text { if }|c|>c_{1} \\
\text { if }|c|=c_{1} \\
\text { if }|c|<c_{1}\end{array}$ & 4 \\
\hline$f_{1}=0$ & $\bigcup_{2}^{\mathbb{S}^{1}}\left(\mathbb{S}^{3} \backslash \mathbb{S}^{1}\right)$ & 15 & $\begin{array}{l}\bigcup_{2} \mathbb{S}^{1} \times \mathbb{R} \\
\bigcup^{1} \mathbb{S}^{1} \times \mathbb{R}\end{array}$ & $\begin{array}{l}\text { if } c \in \mathbb{R} \backslash\{0\} \\
\text { if } c=0\end{array}$ & 16 \\
\hline$f_{1}<0 \leq 2 h$ & $\bigcup_{2}\left(\mathbb{S}^{3} \backslash \mathbb{S}^{1}\right)$ & $15^{*}$ & $\begin{array}{l}\bigcup_{2}^{4} \mathbb{S}^{1} \times \mathbb{R} \\
\bigcup \mathbb{S}^{1} \times \mathbb{R}\end{array}$ & $\begin{array}{l}\text { if } c \in \mathbb{R} \backslash\{0\} \\
\text { if } c=0\end{array}$ & $16^{*}$ \\
\hline $2 h<0<f_{2}$ & $\left(\mathbb{S}^{3} \backslash \mathbb{S}^{1}\right) \bigcup \mathbb{S}^{2} \times \mathbb{S}^{1}$ & 14 & $\begin{array}{c}\mathbb{S}^{1} \times \mathbb{R} \\
\mathbb{S}^{1} \bigcup \mathbb{S}^{1} \times \mathbb{R} \\
\mathbb{S}^{1} \times \mathbb{R} \bigcup \mathbb{S}^{1} \times \mathbb{S}^{1}\end{array}$ & $\begin{array}{l}\text { if }|c|>c_{1} \\
\text { if }|c|=c_{1} \\
\text { if }|c|<c_{1}\end{array}$ & 13 \\
\hline$f_{2}=0$ & $\left(\mathbb{S}^{3} \backslash \mathbb{S}^{1}\right) \bigcup \mathbb{S}^{1}$ & $8^{*}$ & $\begin{array}{c}\mathbb{S}^{1} \times \mathbb{R} \\
\mathbb{S}^{1} \times \mathbb{R} \bigcup \mathbb{S}^{1}\end{array}$ & $\begin{array}{l}\text { if } c \in \mathbb{R} \backslash\{0\} \\
\text { if } c=0\end{array}$ & $9^{*}$ \\
\hline$f_{2}<0$ & $\mathbb{S}^{3} \backslash \mathbb{S}^{1}$ & 8 & $\mathbb{S}^{1} \times \mathbb{R}$ & if $c \in \mathbb{R}$ & 9 \\
\hline
\end{tabular}

TABLE 4. The invariant set $I_{h}$ and its foliation by $I_{h c}$ for $K>0$ and $f_{1}<2 h<f_{2}$. The $15^{*}$ in the column $g^{-1}(h)$ indicates that the surface $g^{-1}(h)$ is the one of Figure 15 but now the two surfaces of Figure 15 do not share a common point. The $16^{*}$ in the column $I_{h} / \mathbb{S}^{1}$ indicates that the foliation of $I_{h}$ by the invariant sets $I_{h c}$ is the same as the foliation in Figure 16 without identifying the two central axes of both solid tori. 


\section{ACKNOWLEDGEMENTS}

This work is supported by the Ministerio de Economía, Industria y Competitividad, Agencia Estatal de Investigación within the grant MTM201677278-P (FEDER), the Agència de Gestió d'Ajuts Universitaris i de Recerca grant 2017 SGR 1617, and the European project Dynamics-H2020MSCA-RISE-2017-777911. The second author is partially supported by FCT/Portugal through the project UID/MAT/04459/2013. The third author is partially supported by Fondecyt project 1180288.

\section{REFERENCES}

[1] R. Abraham and J.E. Marsden, Foundations of Mechanics, Benjamin, Reading, Massachussets, 1978.

[2] V.I. Arnold, Mathematical Methods of Classical Mechanics, Springer-Verlag, 1978.

[3] R.A. Buckingham, The classical equation of state of gaseous Helium, Neon and Argon, Proc. R. Soc. Lond. Ser. A. Math. Phys. Sci., 168, 1938, 264-283.

[4] V.I. Arnold, V.V. Kozlov and A.I. Neishtadt, Dynamical Systems III, Enyclopaedia of mathematical sciences, Springer-Verlag, Berlin, 1978.

[5] M.W. Hirsch, Differential Topology, Graduate Texts in Math., 33, SpringerVerlag, New York, 1976.

[6] J. Lennard-Jones, Cohesion, J. Proc. Phys. Soc., 43, 1931, 461-485.

[7] J. Llibre And A. Nunes, Separatrix surfaces and invariant manifolds of a class of integrable Hamiltonian systems and their perturbations, Memoirs of the Amer. Math. Soc., 513, 1994.

[8] K.R. Meyer, G.R. HAll And D. Offin, Introduction to Hamiltonian dynamical systems and the $N$ - body problem, Second Edition, Springer, 2009.

[9] V. Mioc, E. Popescu And N.A. Popescu, Grous of symmetries in LennardJones-type-problems, Rom. Astron. J., 18, 2008, 151-166.

[10] E. Popescu, Equilibrium points in a Buckingham type problem, Rom. Astron. J., 25, 2015, 149-156.

[11] E. Popescu AND D. Pricopi, Global flow in the generalized Buckhingham's twobody problem, Astrophys Space Sci., 362, 2017, 78-85.

[12] D. Pricopi And E. Popescu, Phase-space structure of the Buckhingham's twobody problem, Astrophys Space Sci., 361, 2016, 190-196.

${ }^{1}$ Departament de Matemàtiques, Universitat Autònoma de Barcelona, 08193 Bellaterra, Barcelona, Catalonia, Spain

E-mail address: jllibre@mat.uab.cat

2 Departamento de Matemática, Instituto Superior Técnico, Universidade de Lisboa, Av. Rovisco Pais 1049-001, Lisboa, Portugal

E-mail address: cvalls@math.ist.utl.pt

${ }^{3}$ Departamento de Matemática, Facultad de Ciencias, Universidad del BíoBío, Concepción, Chile

E-mail address: clvidal@ubiobio.cl 